\title{
Vom Katastrophenverwaltungsrecht zu Thomas Hobbes - Vier Szenarien über Gesellschaft, Staat und Recht bei der Katastrophenfolgenbewältigung
}

Katastrophen gibt es viele. Daher kann nicht erwartet werden, dass einheitliche Reaktionsmuster auf sie bestehen. Im Folgenden geht es weder um die Unzahl persönlicher Beziehungsdramen noch um die Verheerungen durch gewaltförmige Auseinandersetzungen im Gefolge von »failed states « oder um die Untaten von Terroristen oder um die Auswirkungen von ganz legalen Börsencrashs in der globalisierten Weltökonomie, sondern um den Umgang mit katastrophalen Naturereignissen durch Gesellschaft, Staat und Recht. Katastrophen zeichnen sich durch akute Zuspitzungen geballter Schadenshäufungen aus. Dies verschärft ihre Wirkung in der öffentlichen Wahrnehmung gegenüber den Risiken, die sich in schleichenden Prozessen realisieren und dabei leicht als undramatisch verharmlost werden können. Obwohl sich die umweltpolitische Diskussion von Risiken lange Zeit um die sicherheitstechnische Fiktion des »größt anzunehmenden Unfalls « (GAU) fokussierte ${ }^{1}$, musste sie doch nach und nach zur Kenntnis nehmen, dass sich die meisten technisch induzierten Risiken in anderen Verlaufsformen als in abrupten Zerstörungen realisieren. Der Schwund der Artenvielfalt steht für diesen Typus der »schleichenden Katastrophe«.

Allerdings können auch Naturereignisse zu plötzlichen Schadensereignissen mit unermeßlichem Umfang führen. Dabei herrscht in der Katastrophenforschung die Auffassung vor, dass auch in der Natur schon die kleinsten Ereignisse unabsehbare Folgen haben können ${ }^{2}$. Deshalb läßt sich weder ihr Eintritt räumlich und zeitlich noch ihre Schwere präzise vorhersagen. Die Gesellschaft ist daher mit der Kontingenz ihres Eintritts konfrontiert. Zudem sind entwickelte Gesellschaften offensichtlich vielen von Naturprozessen induzierten Katastrophen nur noch am Rande ausgesetzt, die für agrarische Gesellschaften eine dominierende Rolle spielen. Biblische Plagen wie etwa von Heuschrecken und Dürre stellen für sie nur noch eine allegorische Gefahrenquelle dar. Waldbrände sind bisher nur selten bis in die Zentren der modernen Zivilisation vorgedrungen. Die traditionellen Seuchen sind eingedämmt. Die neuen hofft man solange von den Zentren der entwickelten Welt fernhalten oder wenigstens in Subkulturen abdrängen zu können, bis wirksame Therapeutika entwickelt worden sind. Es sind im Wesentlichen die mechanischen Gewalten von Erbeben, Vulkanausbrüchen, Wind und Wasser, die als Katastrophen wahrgenommen werden, weil sie schlagartig immense Schäden verursachen. Sie können nicht nur menschliches Leben in beträchtlicher Zahl

1 Vgl. dazu Charles Perrow, Normale Katastrophen. Die unvermeidlichen Risiken der Großtechnik, Frankfurt/Main 1988; Patrick Lagadec, Das große Risiko. Technische Katastrophen und gesellschaftliche Verantwortung, Nördlingen 1987.

2 Vgl. dazu Mark Buchanan, Das Sandkorn, das die Erde zum Beben bringt. Dem Gesetz der Katastrophen auf der Spur oder warum die Welt einfacher ist als wir denken. Frankfurt/ Main 2001. 
fordern und Sachgüter zerstören, sondern insbesondere auch die technische Infrastrukturen beschädigen, die als Nervenstränge von Mobilität und Kommunikation für das Funktionieren von Wirtschaft und Gesellschaft entwickelter Gesellschaften unerlässlich sind. Werden sie unterbrochen, fällt nicht nur die unmittelbar von der Naturkatastrophe betroffenen Region in Agonie, weit darüber hinaus kann das wirtschaftliche und soziale Leben nachhaltig beeinträchtigt werden. Doch damit nicht genug. Auch der Katastrophenschutz verliert die technische Basis seiner Einsatzfähigkeit.

Mit einer Katastrophe ist nicht alles aus, sondern es beginnen erst die Herausforderungen. Sie bedeutet nicht den Weltuntergang, sondern bleibt ein singuläres, lokal oder zumindest regional begrenztes Ereignis. Erst die Begrenztheit der Zerstörungen ermöglicht es, dass Hilfe überhaupt möglich und eingefordert wird. Schlechthin ubiquitäre und globale Zerstörungen erfassen auch die potentiellen Hilfskräfte. Naturkatastrophen ereignen sich in Gesellschaften, die den sozialen Naturzustand überwunden haben. Die Natur selbst kennt keine Katastrophen. Naturereignisse haben für eine Gesellschaft katastrophale Folgen, wenn sie ihre natürlichen, technischen, baulichen und infrastrukturellen Grundlagen zerstören und damit auch die wirtschaftliche und soziale Reproduktion erfassen. Dabei sind es wiederum die spezifischen Raumnutzungsansprüche der modernen Gesellschaft, die Prozesse der Urbanisierung und Suburbanisierung, ihre Präferenzen für risikobehaftete Formen des Bauens sowie ihre sensiblen Netze von Mobilität und Kommunikation, die sich schadenserhöhend auswirken.

Für die Gesellschaft ist es nicht nur wichtig, die Gründe des Entstehens von Naturereignissen nachvollziehen zu können, sondern noch mehr Maßnahmen zur Vermeidung, Begrenzung und Kompensation ihrer katastrophalen Folgen zu treffen. Katastrophen setzen Diskontinuitäten. Sie bedeuten nicht das Ende, sondern fordern Folgenbewältigung. Aber auch die Folgenbewältigung folgt keinem festem Schema. Ähnliche Naturereignisse können zu ganz unterschiedlichen Schadensbildern führen und vergleichbare Schäden können völlig unterschiedliche politische und gesellschaftliche Folgewirkungen auslösen. Der gesellschaftliche Umgang mit Katastrophen ist nicht weniger kontingent als Muster des Entstehens von Naturereignissen, die katastrophale Folgen haben. Im Gegensatz zum plötzlichen Eintritt der schadenbringenden Naturereignisse muß sich die Gesellschaft mit ihnen in einem zeitlich gestreckten Rahmen antizipatorischer, akuter, nachsorgender und kompensatorischer Katastrophenbewältigung auseinandersetzen. Dies weist auch dem Recht unterschiedliche Rollen und Funktionen zu. Die sozialen Formen der Antizipation von und der Reaktion auf Naturkatastrophen lassen sich daher am ehesten durch Szenarien beschreiben. Naturkatastrophen stellen die Organisationsfähigkeit einer Gesellschaft auf die Probe und testen ihre Potentiale an gesellschaftlicher Solidarität. Die ersten beiden Szenarien befassen sich mit den Bedingungen erfolgreicher Katastrophenbewältigung, die beiden letzten skizzieren unter dem Eindruck des Hurrikans »Katrina« die Folgen des Scheiterns. Im Glücksfall beweist sich die Dichte des sozialen Zusammenhalts. Die Akteure können die politische Prämie des erfolgreichen Krisenmanagements einstreichen. Der Mißerfolg bedeutet Verlust an politischer Legitimität. Im Unglücksfall droht der Rückfall in den gesellschaftlichen Naturzustand des Kampfes aller gegen alle. 


\section{Szenario: Die vermiedene Katastrophe}

Das Szenario der vermiedenen Katastrophe verläuft weithin undramatisch und unspektakulär: Die Erde bebt und die Gebäude halten stand; die Lava strömt, bleibt aber in den vorausberechneten Bahnen; die Sturmflut prallt auf die Deiche, wird aber von ihnen aufgehalten. Die Maßnahmen des vorbeugenden Katastrophenschutzes haben gegriffen. Mit kaum verhohlener Enttäuschung verlassen die Scharen der Katastrophenbeobachter ihre Plätze. Dieses Szenario setzt einen langen Vorlauf des »beharrlichen Bohrens dicker Bretter « (Max Weber) voraus, in dem die Maßnahmenbündel des vorbeugenden Katastrophenschutzes konzipiert, umgesetzt und einsatzbereit gehalten werden.

Vorbeugender Katastrophenschutz ist ein voraussetzungsreiches Projekt. Wir kennen die allgemeinen Vorbedingungen, aus denen heraus Flüsse über die Ufer treten, Feuer sich in ausgetrockneten Wäldern entzündet, Hurrikans, Tornados und Sturmfluten entstehen, Magma aus Vulkanen herausgeschleudert wird oder die Erde zu beben anfängt. Naturereignisse können allerdings nur begrenzt an ihrer Wurzel bekämpft werden. Die Gesetze der Tektonik lassen sich nicht beeinflussen, Sturmfluten und Starkregen können nicht polizeilich verboten werden. Die Natur entzieht sich daher der Regulation nach Maßgabe des Verursacherprinzips. Dies beginnt sich in dem Maße zu ändern, wie die technische Zivilisation die Naturprozesse selbst beeinflusst. Setzt die moderne Zivilisation klimaverändernde Gase frei, fällt die kausale Zurechnung der damit verbundenen Folgen auf sie zurück. Die avisierte Klimakatastrophe ließe sich in der Tat an ihren gesellschaftlichen Wurzeln bekämpfen, wenn diese denn nicht so weit verzweigt wären, dass sie sich dem Entscheidungsfeld politischer Organisationen entzögen.

Der vorbeugende Katastrophenschutz ist daher auf die Eindämmung der Folgen von nicht beeinflussbaren Naturereignissen verwiesen. Er kann dabei den harten Weg des technischen Widerstandes über stärkere Bewehrungen und tiefere Gründung von Gebäuden, Eindeichungen von Küsten, Verbauungen und Kanalisierungen von Flüssen oder den sanfteren Weg des angepassten Bauens oder des Schaffens von Retentionsräumen einschlagen, alle Maßnahmen des Katastrophenschutzes verlangen gleichermaßen vorsorgende, flächendeckende und systemisch angelegte Vorkehrungen. Sie bleiben dabei mit einer doppelten Ungewissheit belastet. Sie sind zugleich Zweifeln ausgesetzt, ob sie übermäßig oder unzureichend dimensioniert sind. Da sich Zeitpunkt, Ort und Schwere von Naturereignissen, aus denen für die Gesellschaft katastrophale Folgen erwachsen können, nicht hinreichend genau prognostizieren lassen, können die vorbeugenden Maßnahmen nicht präzise justiert werden. Auch wenn sie großzügig dimensioniert, auf Vorrat angelegt und mit hohen Risikoaufschlägen versehen werden, bleibt ein ungewisser Rest an nicht abgedeckten Risiken.

Diese Form des vorbeugenden Katastrophenschutzes ist eine Aufgabe der öffentlichen Daseinsvorsorge und damit eine teuere Gemeinlast. Sie erweist sich als weithin alternativlos. Katastrophenvorsorge für eine ganze Region ist auf dem Markt nicht zu kaufen. Sie muß vielmehr gegenüber seinen viel enger dimensionierten und kurzfristiger angelegten Risikokalkülen durchgesetzt werden. Private Vorsorge durch Versiche- 
rungen greift erst nach Schadenseintritt ein. Sie ist überdies auf Sachschäden begrenzt. Vorbeugender Katastrophenschutz ist daher die Domäne des Öffentlichen Rechts. Elemente finden sich in einer Vielzahl von Rechtsvorschriften der Raumordnung, der Bauleitplanung und der Bauordnung oder des Gewässerschutzes verstreut und nicht zuletzt in dem unzähligen Bestand der privaten Normung. Sie belasten das private Eigentum und sind als Ausfluß seiner Situationsgebundenheit grundsätzlich hinzunehmen $^{3}$.

Eine solche administrative Risikovorsorge gegenüber nur abstrakt denkbaren Ereignisse ist nicht leicht zu vermitteln. Sie setzt ein politisches Vorverständnis voraus, das Vorsorge gegen Risiken als grundlegende staatliche Aufgabe anerkennt und sie nicht der privaten Initiative überlässt. Das Grundgesetz setzt hier mit dem an den Staat adressierten Auftrag, sich »schützend und fördernd vor die Grundrechte zu stellen ${ }^{4}$, eine wichtige Markierung, gesteht ihm gleichzeitig allerdings auch Einschätzungs-, Wertungs- und Gestaltungsspielräume $\mathrm{zu}^{5}$. Diese Schutzpflichten sind als Reaktion auf Risiken entwickelten worden, die von der Gesellschaft selbst ausgehen. Gegenüber Naturereignissen, wie etwa dem Schutz vor Hochwasser, haben sie zwar nicht als subjektiv-rechtliche Rechtsansprüche auf staatliche Katastrophenvorsorge, wohl aber als objektiv-rechtliche Verpflichtung für das Allgemeinwohl Anerkennung gefunden 6 .

Auch für New Orleans kam die Katastrophe nicht aus heiterem Himmel. Über ihren Verlauf gab es bis ins Detail gehende Vorhersagen. Sie wurden nicht nur von Experten des Küstenschutzes diskutiert, sondern waren auch von der Lokalpresse verbreitet worden. Es gilt als sicher, dass sich die katastrophalen Folgen des Hurrikans »Katrina« hätten vermeiden lassen, wären nur die notwendigen Maßnahmen getroffen worden. Die erforderlichen investiven Mittel standen jedoch nicht zur Verfügung. Der Etat des Küstenschutzes war um ein Drittel gekürzt worden. Die eigentlich zuständige Katastrophenschutzbehörde FEMA wurde in eine Terroristenabwehrbehörde umdefiniert. Dies war möglich, weil zwar der Kampf gegen den Terror als basale Herausforderung des politischen Systems der Vereinigten Staaten gilt, während dort die Vorsorge gegen Risiken für Leib, Leben und Eigentum primär als Privatangelegenheit betrachtet wird.

\section{Szenario: Die optimistische Tragödie}

Dieses Szenario ist voller Dramatik. Es beginnt tragisch, endet aber mit einem für die Gesellschaft letztlich versöhnlichen Ausklang: die Deiche brechen, die größten Zerstörungen können aber gerade noch vermieden werden. In ihm gibt es eine heroische

3 Vgl. dazu jüngst Thomas Bosecke, Vorsorgender Küstenschutz und Integriertes Küstenzonenmanagement (IKZM) an der deutschen Ostseeküste. Strategien, Vorgaben und Defizite aus Sicht des Raumordnungs-, Naturschutz- und europäischen Habitatschutzrechts sowie des Rechts der Wasserwirtschaft. Heidelberg - New York 2005, S. 486 ff.

4 BVerfGE 39, $1<41>$; 49, $89<141>$; 53, $30<57>$; 56, $54<73>$; 77, $170<214>, 79,174$ $<201>$.

5 BVerfGE 77, $170<237>$.

6 Vgl. Rüdiger Breuer, Hochwasserschutz im geltenden und zukünftigen Recht, Köln 1999, S. 50. 
Hauptrolle. Sie ist mit den Helfern besetzt. Ihre Aktionen beziehen sich auf ein nicht weniger heroisches Umfeld der Betroffenen, die alles zu ihrem Schutz und zur Rettung anderer getan haben, deren Kräfte aber schließlich schwinden, so dass sie selbst von den professionellen Kräften des Katastrophenschutzes vor größeren Schaden bewahrt werden müssen.

Erweisen sich die vorsorgenden Maßnahmen als unzureichend, ist akute Gefahrenabwehr gefordert. Der Katastrophenschutz muß Personen bergen, Brände löschen, Keller leerpumpen, Gebäude vor Einsturz sichern, Deiche verteidigen, nicht mehr haltbare Stellungen aufgeben, Räume opfern, Auffangstellungen beziehen. Dies mag militärisch klingen, hat jedoch eine durch und durch zivilgesellschaftliche Ausrichtung. Der Katastrophenfall bezeichnet einen unpolitischen Ausnahmezustand der »Risikogesellschaft«. In ihm schlägt zwar die »Stunde der Exekutive«, aber nicht des Staatsschutzes. Dem »Katastrophenverwaltungsrecht ${ }^{7}$ geht es nicht um die Bewahrung des politischen Systems, sondern um die Güter der Zivilgesellschaft. Dabei hängt der Erfolg der Einsatzkräfte weniger von ihrer schlichten Zahl als vielmehr von ihrer Ausbildung und der Verfügbarkeit von geeignetem technischen Gerät und noch mehr von einer funktionierenden Katastropheninfrastruktur ab, die erst deren Mobilität und Kommunikation zu gewährleisten vermag. Doch damit nicht genug. Die professionellen Kräfte der Katastrophenwehr bestehen aus einer Vielzahl von Organisationen: Feuerwehr, Rettungswesen, Technisches Hilfswerk und last not least der Bundeswehr. Ihre Aktionen müssen koordiniert werden. Amtshilfe wird so zum allgemeinen normativen Leitprinzip des Katastrophenschutzrechts ${ }^{8}$. Übersteigt die Katastrophen das Vermögen der nationalen Kräfte, kommt die Koordination der internationalen Hilfe als zusätzliche Herausforderung hinzu.

Darin offenbaren sich zwei entscheidende Voraussetzungen: die akute Katastrophenabwehr benötigt einen langen strategischen Vorlauf an institutionalisierter Organisationsvorsorge, ihr taktischer Einsatz verlangt Kooperation und Koordination. Dies ist in einem föderal segmentierten und funktionell ausdifferenzierten politischen System keine Selbstverständlichkeit. Es ist zurecht darauf hingewiesen worden, dass sich hier die Funktion des Rechts weniger auf materiell-rechtliche Gewährleistungen als vielmehr auf Organisation, Verfahren und Zuständigkeiten konzentriert ${ }^{9}$. Es ist prozedural dimensioniert. Im weiteren enthält das Recht zwar weitreichende Eingriffsbefugnisse, kann aber nicht ernsthaft Sicherheit und Schutz im materiellen Sinne versprechen, sondern lediglich die Mobilisierung aller verfügbaren Mittel des Katastrophenschutzes. Doch auch diese prozeduralen Leistungen haben eine vorsorgende Komponente. Sie müssen vor dem Katastrophenfall getestet, eingeübt und evaluiert sein. Diese Sichtachse auf die professionellen Helfer lässt außer Betracht, dass entscheidende Beiträge zur Eindämmung der Katastrophenfolgen aus der Gesellschaft

7 Rolf Stober/Sven Eisenmenger, Katastrophenverwaltungsrecht - Zur Renaissance eines vernachlässigten Rechtsgebietes, NVwZ 2005, $121 \mathrm{ff}$.

8 Stober/Eisenmenger, NVwZ 2005, 123.

9 Vgl. dazu Hans-Heinrich Trute, Katastrophenschutzrecht - Besichtigung eines verdrängten Rechtsgebietes, KritV 2005, 342 ff. 
selbst kommen. Von der Aktivität der Betroffenen hängt es entscheidend ab, in welchem Umfang Schadensbegrenzung erreicht werden kann. Das expertokratische Modell des Katastrophenmanagements bedarf einer zivilgesellschaftlichen Ergänzung. Ihre beiden zentralen Faktoren sind Eigeninitiative und nachbarschaftliche Solidarität. Deren Mobilisierung liegt weit außerhalb der Reichweite eines die Rechtsfigur der Amtshilfe operationalisierenden »Katastrophenverwaltungsrechts «.

So endet der erste Akt dieses Szenarios mit dem Bild der erschöpften, aber erfolgreichen Einsatzkräfte inmitten von gleichfalls erschöpften, aber schon wieder Hoffnung fassenden Betroffenen. Trotz großen Leids und vieler Opfer hat die Gesellschaft den Herausforderung durch die Naturgewalten dank ihrer vereinten Anstrengungen standgehalten. Das Finale wird gekrönt, wenn sich aus der Vielzahl der Helfer eine Lichtgestalt herauskristallisiert, die in den Medien als Katastrophenmanager gefeiert werden kann. Der erfolgreiche Deichgraf hat sich für höhere Aufgaben empfohlen. Der zweite Akt dieses Szenarios wird der Öffentlichkeit zumeist nur in seiner ersten Szene dargebracht. Sie zeigt die Mobilisation der Kräfte zum Wiederaufbau. Darin liegt der eigentliche Test der Organisationsfähigkeit der Gesellschaft. Der Auftakt zum Wiederaufbau beginnt idealtypisch mit dem Duett gesellschaftlicher und staatlicher Hilfsversprechen. Dem Recht ist hier keine tragende Rolle zuerkannt. Die Medien machen sich zum Anwalt der Betroffenen. Sie organisieren unter Mitwirkung der von ihnen lebenden Personen des öffentlichen Lebens Spendenaktionen. Die Gesellschaft der Individuen hat hier hinlänglich unter Beweis gestellt, dass die Opfer von Katastrophen nicht auf sich allein gestellt sind. Sie hat vielmehr ein überbordendes Potential an gesellschaftlicher Solidarität demonstriert. Spenden kompensieren die nichtversicherten oder nichtversicherbaren Risiken. Ihnen treten die staatlichen Hilfen zur Seite, mit denen gezielt und regelgebunden Ersatz für vernichtete Sachgüter geschaffen werden soll. Mit diesem Bild, das längst verloren geglaubte Qualitäten gesellschaftlicher Synthesis wieder erweckt, endet zumeist die Katastrophenberichterstattung.

Gleichwohl werden die entscheidenden gesellschaftlichen Leistungen erst im Anschluss daran abgefordert, wenn es gilt, den Wiederaufbau tatsächlich ins Werk zu setzen. Auch hier hängt das Gelingen entscheidend von der Initiative der betroffenen Individuen ab. Der Selbsthilfe ist es weitgehend überlassen, wann mit der Instandsetzung und dem Wiederaufbau begonnen wird. Aber auch die Verteilung der Wiederaufbauhilfen benötigt ein zuverlässiges institutionelles Widerlager, um rasch und zielgerichtet zu wirken und um Missbrauch zu minimieren. Dies gilt um so mehr für die Wiederherstellung der zerstörten öffentlichen Infrastrukturen. Hier betritt das Recht wiederum die Bühne. Es schärft die Bedingungen für die Vergabe von Darlehen und verlorenen Zuschüssen zum Wiederaufbau und sanktioniert deren Missbrauch. In seinen Strukturen gehört es nicht mehr zum »Katastrophenverwaltungsrecht «, sondern signalisiert als klassisches Verwaltungsrecht das Ende des Ausnahmezustandes und den Eintritt in die Normalität.

Greift das Räderwerk der organisierten Wiederaufbauhilfen, kann schließlich die positive Bilanz gezogen werden, die zerstörten Dörfer und Städte seien schöner denn je wiedererstanden. So endet die optimistische Tragödie in dem Schluss, dass Gesellschaft erfolgreich den »ungeregelten Gewalttätigkeiten der Natur« (Fichte) zu wider- 
stehen vermochte. Das Maß, von dem sich die Gesellschaft von diesem Gefühl blenden lässt und darüber das Nachdenken über die Verbesserung der Vorsorge gegen Katastrophen vergisst, bestimmt über die Wahrscheinlichkeit und Intensität ihrer Wiederkehr.

\section{Szenario: Staatsversagen als Katastrophe höherer Ordnung}

Hier dominiert nicht der Heroismus einer Gesellschaft, die erfolgreich den Herausforderungen der Naturgewalten widersteht, sondern die Unfähigkeit ihres politisch-administrativen Systems, die erforderlichen Organisationsleistungen zu erbringen. Das Szenario der Katastrophe höherer Ordnung demonstriert somit dessen Pathologien zum und die persönliche Inkompetenz der verantwortlichen Akteure im Katastrophenmanagement. Es ist zu traurig, um es theatralisch in die Form einer Tragödie oder Komödie zu bringen. Das Staatsversagen wird von der Öffentlichkeit in der Regel mit hämischen Kommentaren quittiert. Es eignet sich eher als Gegenstand einer Satire oder einer Farce.

Katastrophenschutz kann Sicherheit nicht ernsthaft gewährleisten, sondern lediglich die Organisation von Hilfe. Er wird von einem protestantischen Ethos getragen. Seine basale Aufgabe ist bereits dann erfüllt, wenn sich die Helfer nach Kräften bemühen. Ihnen kann kein ernsthafter Vorwurf gemacht werden, wenn es trotz aller Anstrengungen nicht gelingt, den Deich zu halten, Sachgüter zu bewahren oder Leben zu retten. Um so schwerer wiegt die Kritik, kein Rettungstrupp sei vor Ort gewesen, im Meldewesen habe Chaos geherrscht, die Einsatzkräfte seien anderswo gebunden gewesen oder hätten sich gegenseitig blockiert, die Einsatzleitung habe sich im Kompetenzwirrwarr verstrickt, auch mehrere Tage nach der Katastrophe seien keine zuverlässigen Zahlen über die Opfer vorhanden, Leichen könnten weder identifiziert noch bestattet werden. Auch der zweite Akt der Katastrophenbewältigung kann entgleisen, wenn bekannt wird, dass die reichlich geflossenen Spenden des In- und Auslandes in anonymen Kanälen versickert sind. Sind die lokalen Apparate zur Verteilung der Mittel zum Wiederaufbau durch die Auswirkungen der Katastrophe selbst gestört oder waren sie bereits vorher defizitär, ist das Versagen der administrativen Hilfen vorprogrammiert. Solche Pathologien der Katastrophennachsorge sind der ultimative Beweis für Staatsversagen.

Entsprechende Nachrichten sind nicht ungewöhnlich. Sie begleiten fast immer als Parallelhandlung das Szenario der optimistischen Tragödie. Entscheidend ist, dass die Belege für das Vermögen der Katastrophenbewältigung letztlich Überhand über die Indizien für das Unvermögen erlagen. Aber auch bei einem anderen Ausgang ist noch eine Wendung mit optimistischen Ausblick möglich. Gerade wenn die Organisationsleistung unzureichend ist, entwickelt sich aus dem Organisationsversagen ein Maßstab für Kritik. Sie erinnert daran, was hätte getan werden müssen. Sie thematisiert Verantwortung und fordert strukturelle und personelle Konsequenzen. Ein solcher Sturm nach dem Sturm kann kathartische Wirkung haben. Wie in der Krise liegt auch in der Katastrophe die Chance zur Umbesinnung. Werden die eingeforderten Konsequenzen rechtzeitig und in hinreichender Schärfe gezogen, besteht die Chance, dass das politische System Lernfähigkeit beweist und sich von den Verlusten an Legitimität erholen kann. 
Die Reaktionsmuster auf den Hurrikan »Katrina« weisen in andere Richtung. Zunächst folgten die Strategien des Katastrophenschutzes nicht dem Muster der antizipatorischen Folgenbewältigung. Als ihre Defizite angesichts der zu prognostizierten Schwere des Sturms sichtbar wurden, kulminierten die Vorkehrungen der Einsatzleitung nicht in der Verstärkung der Deiche vor Ort, sondern in der Aufforderung, New Orleans zu verlassen. Die Präferenz für die individuelle Vorsorge verlängerte sich somit in die Form der Bewältigung akuter Gefahren. Der schlichte Appell »rette sich, wer kann « vernachlässigte allerdings, dass sich in einer Stadt ohne funktionierenden öffentlichen Verkehr nur diejenigen rechtzeitig in Sicherheit bringen konnten, die im Besitz eines Pkws waren. Dies traf in der als »automobil« apostrophierten Gesellschaft für etwa 100.000 Menschen nicht zu. Zudem ließ die Verwaltung ihre Flotte von Schulbussen, mit denen diese Personen hätten evakuiert werden können, schlichtweg in den Fluten untergehen. Dies ist nicht nur ein Zeichen für einen »schlampigen« Staat $^{10}$, der die Daseinsvorsorge für seine Bürger vernachlässigt. Wurden in den Fluten alle diejenigen zurückgelassen, die aus dem Selbstbildnis der amerikanischen Gesellschaft traditionell ausgeblendet sind, spülten sie folgerichtig die Strukturen einer durch die Verwerfungen von »class, race and poverty « geschiedenen Gesellschaft frei. Aber auch hier vermag New Orleans noch eine weitere Nuance zu setzen. Die lokale Verwaltung musste vier Wochen nach dem Hurrikan gleichsam politische Insolvenz anmelden, da sie nicht mehr über die erforderlichen Mittel zur Bezahlung ihres Personals verfügt. Sie wird offensichtlich von ihrem Bundesstaat ebenso wie von der föderativen Gewalt nicht als Empfänger der inzwischen reichlich zur Verfügung gestellten Hilfsgelder in Betracht gezogen. Auch dies reiht sich in das Bild eines politischen Organisationsversagens ein. Noch brisanter könnte sich allerdings die Verlängerung des Organisationsversagens in personelle Inkompetenz erweisen. So etwa, wenn Nachrichten, dass der verantwortliche Leiter der FEMA sich für dieses Amt durch seine frühere Präsidentschaft über einen Verband für Pferdezucht empfohlen hat, und Bilder eines ohnmächtigen und jeder Selbstkontrolle verlustigen Bürgermeisters, einer wegen Überforderung weinenden Gouverneurin sowie eines abwesenden und in der Sache wenig anteilnehmenden Präsidenten längere Zeit die Medien beherrschen. Auch hier hält das demokratische Systems die Verfahren vor, wie die entsprechenden Konsequenzen zu ziehen sind. Sie wurden bereits vehement eingefordert. Allerdings gibt es auch hier ein worst-case-Szenario. Es kulminiert darin, dass keine organisatorischen Folgerungen getroffen werden und die Öffentlichkeit mit personellen Bauernopfern befriedigt wird. Dies kann die Verantwortlichen retten, allerdings nur auf Kosten des Basisvertrauens in das politische System.

\section{Szenario: Der Rückfall in den gesellschaftlichen Naturzustand}

Die vom Hurrikan »Katrina« unmittelbar verursachten Schäden sind vergleichsweise gering. Etwa 1000 Tote sind zu beklagen. 150.000 Häuser sollen zerstört und etwa 200.000 Menschen obdachlos geworden sein. Ein Erdbeben von 1995 forderte in der

10 Jedediah Purdy, Eine Lehrstunde der Wölfe, in: Die Zeit 37/2005, S. 3. 
japanische Stadt Kobe 5000 Tote und 30000 Verletzte. 100.000 Gebäude wurden zerstört, 300.000 Einwohner wurden obdachlos ${ }^{11}$. Es erschütterte das politische System Japans nicht. Selbst der Tsunami vom 26.12.2004, der zwar das Versagen des Katastrophenschutzes in den betroffenen Ländern offenbarte, löste keine größeren politischen Turbulenzen aus. Vielmehr entwickelten sich Ansätze, die regionalen Separationsbewegungen in den Wiederaufbau zu integrieren. Im Unterschied dazu hinterließ der Hurrikan »Katrina« zumindest bei europäischen Beobachtern den Eindruck eines aufbrechenden gesellschaftspolitischen Desasters.

Die Katastrophenbekämpfung blieb nicht nur weitgehend aus, ihre verstreuten Kräfte wurden sogar von marodierenden Gangs beschossen. Geschäfte wurden nicht nur aus schierer Not von den Zurückgelassenen geplündert, sondern auch zum Zwecke der Bereicherung ausgeraubt. Ähnlich erging es einigen Hilfskonvois. Ein Drittel der Polizeibeamten meldeten sich am Tage nach dem Sturm nicht mehr zum Dienst. Als sich schließlich die staatliche Ordnungsmacht mit der evakuierten weißen und wohlhabenden Bevölkerung förmlich zurückzog, bemächtigten sich Banden der überfluteten Stadt, deren Aktivitäten früher auf einige Quartiere von New Orleans beschränkt waren. Sie überschritten die virtuellen Grenzen ihrer »no-go-areas« und nutzten das Vakuum der politischer Ordnung, um die letzten Inseln in ihre Gewalt zu bringen, auf denen die Bewohner Zuflucht gesucht hatten, denen es nicht gelungen war, die Stadt rechtzeitig zu verlassen. Die provisorischen Sammelstellen wie der »Superdome« und das Kongresszentrum wurden zur Falle für alle, die sich dorthin gerettet zu haben glaubten. In ihnen etablierten Gangs ihr Regime. Die gesamte Stadt wurde zur »no-goarea « eines lokalen Reiches der unkontrollierten Gewaltsamkeit. Im Halbdunkel der Szenerie von überfluteten und brennenden Gebäuden, desorientierten Personen und Gewalttaten begannen die von Hollywood erfundenen »day-after"-Plots postapokalyptischer Überlebenskämpfe Wirklichkeit zu werden.

Die Naturkatastrophe verlängerte sich in einen politischen und gesellschaftlichen Offenbarungseid. Nicht nur Jedediah Purdy drängte sich die Assoziation eines »Bagdad am Mississippi« auf, wo »die Unfähigkeit, die elementarsten Anforderungen an staatliche Hoheitsausübung zu erfüllen, ein moralisches Debakel angerichtet hat $\aleph^{12}$. Zudem gibt es Anzeichen dafür, dass die staatliche Gewalt die Kontrolle nicht schlechthin in dem durch die Naturgewalten verursachten Chaos verloren hat, sondern sich gar nicht bemühte, sie aufrecht zu erhalten. Die aus New Orleans gesendeten Bilder einer zurückweichenden und gleichsam uninteressierten Staatsgewalt gleichen denen des Einmarsches amerikanischer Truppen in Bagdad, die gleichermaßen gegen Plünderungen und Gewalttaten nicht bereit waren einzuschreiten, wenn ihre unmittelbaren militärischen Ziele nicht berührt waren. Das zum Desinteresse gesteigerte Unvermögen der Ordnungskräfte erweckte nicht nur den Eindruck eines an der Fürsorge für seine notleidenden Bürger gescheiterten Staates, die nicht mehr präsente Staatsmacht verwies vielmehr überdies auf eine »failed society«, in der die Grundanforde-

11 Buchanan, S. 18.

12 Purdy, a.a. O. ; vgl. auch Slavoj Zizek, Flucht aus New Orleans, in FR vom 7. 9. 2005, S. 27 sowie Der Spiegel 37/2005, S. 129. 
rungen des sozialen Zusammenlebens keine Geltung mehr besitzen. Die zivilgesellschaftliche Lebenswelt schien hinweggespült. Der soziale »ground zero« einer gesellschaftlichen Katastrophe wurde sichtbar.

Ist der Gesellschaftsvertrag gekündigt, fällt das Recht auf seine Rudimentalformen zurück: Notwehr und Selbsthilfe. Inmitten von zerstörten Gebäuden, angespülten Überresten der Wohlstandsgesellschaft, Unrat, Kadavern und Leichen versuchten einige, die Reste ihres Eigentums zu schützen. An einem Geschäft im von der Ordnungsmacht aufgegebenen New Orleans war als deutliche Warnung an die vagierenden Banden zu lesen: »I am here, I have a gun« oder in knapper Militanzpoetik »You loot, I shoot«. Angesichts dieses Rückfalles in einen Zustand »aus beständiger Furcht und Gefahr eines gewaltsamen Todes « taucht die Erinnerung an den von Thomas Hobbes beschriebenen Urzustand wieder auf, in dem das menschliche Leben bekanntlich »einsam, armselig, ekelhaft, tierisch und kurz « ist ${ }^{13}$. Sie ruft ins Gedächtnis zurück, dass der gesellschaftliche Naturzustand für eine moderne Gesellschaft die größte aller denkbaren Katastrophen ist.

Allerdings ist die durch den Hurrikan »Katrina« freigesetzte hobbesianische Regression auf das Zentrum der Verwüstungen lokal begrenzt geblieben und war auch dort nur von kurzer Dauer. Es liegt in der Logik des Hobbes'schen Gesellschaftsmodells, dass die Restauration ordnender und sichernder Strukturen nicht mit der Einrichtung einer Notversorgung für die Betroffenen, sondern mit dem Einzug der Nationalgarde begann. Der Leviathan kehrte ohne Schwierigkeiten zurück. Er erzwang die völlige Evakuierung der Stadt. Erst als im sozialen Vakuum seine hinweggespülten law-andorder-Funktionen reetabliert waren, wurden die Deiche zum Lake Pontchartrain geflickt und die Pumpen in Gang gesetzt, um die Stadt Orleans vom Wasser zu befreien. Parallel dazu begannen private Sicherheitsdienste die Quartiere der Wohlhabenden zu sichern. Dies gilt allerdings nicht für die Apparate, die das kompensatorische Katastrophenverwaltungsrecht der Verteilung von Hilfsgütern vor Ort zu exekutieren haben. Vier Wochen nach dem Hurrikan wurde bekannt, dass die Stadt etwa die Hälfte ihrer Beschäftigten mangels verfügbarer Finanzmittel entlassen muss.

\section{Epilog}

Für die amerikanische Gesellschaft, die ohnehin starke Präferenzen für Selbsthilfe hat und auch im Bereich der öffentlichen Sicherheit mehrheitlich dem staatlichen Gewaltmonopol mißtraut, dürften allerdings die Schlaglichter einer hobbesianischen Regression weniger dramatisch als für den europäischen Beobachter sein. Zeigte sich in den Katastrophengebieten von Louisiana, wie dünn die zivilisatorische Kruste moderner Gesellschaften sein kann, und dass die Supermacht mit Strukturen der Dritten Welt durchsetzt ist, belegt der Einsatz der Hilfskräfte am 11. 9. 2005 in den zusammenbrechenden Türmen des New Yorker World Trade Centers, dass sie auch über andere Reaktionsmuster auf katastrophale Ereignisse verfügt. Sie kann im Weiteren darauf ver-

13 Thomas Hobbes, Leviathan oder Stoff, Form und Gewalt eines bürgerlichen kirchlichen Staates, Kap. 13, hrsg. und eingeleitet von Iring Fetscher, Neuwied und Berlin, 1966, S. 96. 
weisen, dass sich ihre Bürger in der Regel solidarisch verhalten. Hilfe für die Betroffenen wurde und wird im Wesentlichen außerhalb der Zone der Verwüstungen geleistet. Hier war es zunächst und vor allem die privat oder von lokalen Gemeinschaften organisierte Solidarität, die den Weg zu den Davongekommenen fand. Sie belegt, dass die amerikanische Gesellschaften auf Gemeinschaften basiert, in denen nicht der Kampf aller gegen alle herrscht. New Orleans gibt daher nicht nur Anlaß zu resümieren, wie wenig die Gewaltpotentiale einer asymmetrischen Gesellschaft domestiziert sind, sondern stärkt auch den Befund, dass sie gleichzeitig mit einem kommunitären Netzwerk sozialer Kohäsion ausgestattet ist. 